\title{
Inferring nonlinear lateral flow immunoassay state-space models via an unscented Kalman filter
}

\author{
Nianyin $\mathrm{ZENG}^{1 *}$, Zidong $\mathrm{WANG}^{2,3}$ \& Hong $\mathrm{ZHANG}^{1}$ \\ ${ }^{1}$ Department of Instrumental and Electrical Engineering, Xiamen University, Fujian 361005, China; \\ ${ }^{2}$ Department of Computer Science, Brunel University London, Uxbridge, Middlesex UB8 3PH, UK; \\ ${ }^{3}$ Faculty of Engineering, King Abdulaziz University, Jeddah 21589, Saudi Arabia.
}

Received January 1, 2016; accepted xx, 2016; published online xx, 2016

\begin{abstract}
This paper is concerned with the problem of learning structure of the lateral flow immunoassay (LFIA) devices via short but available time series of the experiment measurement. The model for the LFIA is considered as a nonlinear state-space model that includes equations describing both the biochemical reaction process of LFIA system and the observation output. Especially, the time-delays occurring among the biochemical reactions are considered in the established model. Furthermore, we utilize the unscented Kalman filter (UKF) algorithm to simultaneously identify not only the states but also the parameters of the improved state-space model by using short but high-dimensional experiment data in terms of images. It is shown via experiment results that the UKF approach is particularly suitable for modelling the LFIA devices. The identified model with time-delay is of great significance for the quantitative analysis of LFIA in both the accurate prediction of the dynamic process of the concentration distribution of the antigens/antibodies and the performance optimization of the LFIA devices.
\end{abstract}

Keywords Lateral flow immunoassay, Biochemical reaction networks, Modelling, unscented Kalman filter

Citation Zeng N Y, Wang Z D, Zhang H. Inferring nonlinear lateral flow immunoassay state-space models via an unscented Kalman filter. Sci China Inf Sci, 2016, xx(x): xxxxxx, doi: xxxxxxxxxxxxxx

\section{Introduction}

In recent years, the lateral flow immunoassay (LFIA) devices have been studied extensively and have become the basis of point-of-care testing (POCT) for clinical diagnostics due conceivably to LFIA's remarkable advantages including rapidness, simplicity, good specificity and sensitiveness [ㅍ, [1, ㅍ]. Owing to these attractive properties, LFIAs have been successfully applied in targeted analytes such as clinical

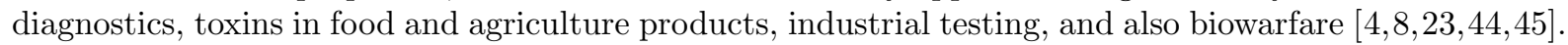
A critical limitation in immunochromatographic assays, however, is that most assays are qualitative (or semi-quantitative) [I] and this, to some extent, has hindered these assays from further applications especially scientific experiments where accurate prediction is required. Therefore, based on the material selection (see e.g. [ㅍ, [3], 30] $)$ ), a number of methods have been introduced for improving the biochemical properties of the strips over the past decade. On the other hand, it is often desirable to increase the relia-

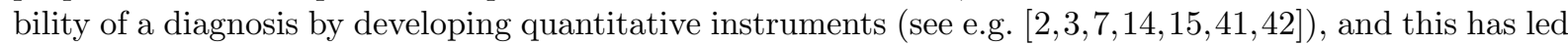
to a growing research interest on developing multidisciplinary approaches to modeling and analyzing the

*Corresponding author (email: zny@xmu.edu.cn) 
LFIA devices in order to optimize immunochromatographic strip performance for the quantification, see e.g. [25, [26, 37-401,43]. The present research of this paper is along the new research line of inferring more accurate models for LFIA state-space models by exploiting more computational appealing algorithms.

A mathematical model for LFIA devices is firstly established by the convection-diffusion-reaction equations in [25, 26] , where the COMSOL software is exploited to demonstrated the process of the biochemical reactions of LFIA. Here, it is worth highlighting that a nonlinear state-space model has been developed in [37-3.9] for sandwich-type LFIA devices. The established model includes the equations for describing the biochemical reaction process of LFIA system and the observation output. Especially, the process of the concentration distribution of LFIA devices is described by the nonlinear state equations, and the available observation signals are modeled by the observation equation including the measurement noises. It should be mentioned that such a model allows us to predict kinetic characteristics and also optimize device performance. Unfortunately, the time-delay which exists among the biochemical reactions of LFIA has not been taken into account in [37-39.] despite the fact that the sample containing the target analytes does need time to flow from the sample pad along the nitrocellulose membrane encountering the detection zone via capillary action. In order to establish a more accurate model for the LFIA, the main purpose of this paper is to further incorporate the inherent time-delays between the biochemical reactions into the modeling process by looking for more efficient algorithms.

A critical issue in inferring nonlinear LFIA state-space models is to search for a justifiable algorithm capable of jointly estimating parameters and states for nonlinear systems with time-delays. So far, we have used the extend Kalman filter (EKF) [37], hybrid EKF and switching PSO (particle swarm optimization) algorithm [3.9] and particle filter [38] for the system identification problems of the nonlinear state-space models. The EKF approach is a suboptimal state estimator which utilizes the Taylor expansion to linearize the nonlinear model and then the traditional Kalman filter (KF) is applied to the linearized model. As such, when the models are highly nonlinear, the EKF approach cannot get good performance. Note that the model of LFIA devices to be developed includes inherent high degree of nonlinearities that would make it ineffective to exploit the KF and EKF approaches. Also, satisfactory usage of the particle filter generally needs a sufficient amount of data so as to the statistical inference, which is not the case for the LFIA data (images) that is typically a short number of time series. In search of a qualified identification algorithm, the unscented Kalman filtering (UKF) [27, 3:3] stands out as an ideal candidate for the LFIA systems that is specialized in handling nonlinearities and incorporating time-delays.

Based on the unscented transform (UT), the UKF algorithm is developed for nonlinear systems as a recursive state estimator. The unscented transform (UT) is a deterministic sampling technique, which utilizes a set of $2 n+1$ sample points (called "sigma points") to estimate the statistics characteristics of the transformed variable. Especially, the sigma points are generated deterministically from a priori mean and covariance of the states. Therefore, both the posterior mean and the covariance of UKF approach are computed directly from these $2 n+1$ sigma points by going through nonlinear transformation [5, [2, [3.3]. Note that the UKF can approximate the posterior mean and covariance for nonlinear systems without any linearization step, but can get second-order or higher order accuracy. However, the EKF algorithm can only obtain first-order accuracy by using the linearization method. In case of high nonlinearities, the UKF algorithm represents a capable alternative to the EKF method that requires accurate evaluation of the Jacobians of the nonlinear functions. Owing to the attractive advantages, the UKF has widely used in many nonlinear systems (see e.g. [5, [12, [17, [24, 272, 32, 33., 35] ) at the cost of relatively high computational cost. Nevertheless, the identification of the LFIA model can be conducted off-line and the computing efforts are really an issue in this case. Hence, in this paper, the UKF algorithm is exploited to identify both the system states and the parameters of the improved LFIA state-space model with time-delay via short available experiment measurement.

It should be pointed out that it is a challenging task to apply the UKF approach in inferring nonlinear LFIA state-space models. This is simply because of the distinguishing features of the LFIA system such as high-degree of nonlinearities, small number of time series, prolonged time-delays between the biochemical reactions as well as noisy observations. It is, therefore, the aim of this paper to infer the nonlinear LFIA state-space models with time-delays via unscented Kalman filtering by overcoming the identified challenges. The contributions of this essay are twofold. 1) An improved nonlinear state-space model is established for the LFIA system where the time-delays which exist among the biochemical reactions are considered in the established model. 2) The UKF algorithm is applied to simultaneously identify the system parameters and states of the LFIA system via short but available time series data and it is shown that the UKF approach achieves satisfactory accuracy for modeling the lateral flow immunoassay since 
no linearization is involved.

The organization of this paper is listed as follows. In Section $\square$, the LFIA format is introduced and the improved model with time delays is proposed for the LFIA system. In Section [1, the UKF method for simultaneously identifying the parameters and the states of the improved LFIA model is conducted. The results of the UKF method for the LFIA system identification are discussed in Section 6 and some indexes are utilized to evaluate the model performance. In the end, conclusion remarks are provided in Section 15 .

\section{Improved LFIA Model with Time-delay and Problem Formulation}

A typical LFIA device format, which can be seen in Fig. $\mathrm{W}$, consists of a surface layer to carry the sample from the sample pad via the conjugate pad along the nitrocellulose membrane encountering the detection zone up to the wicking pad. Quantitative detection of immunocomplexes formed on the test line, that has direct relationship with the concentration of the target analyte in the samples, has been obtained by detecting the reflectance of labeled particles on the test line (signal intensity) via a reader system [QZ, BU] $]$.

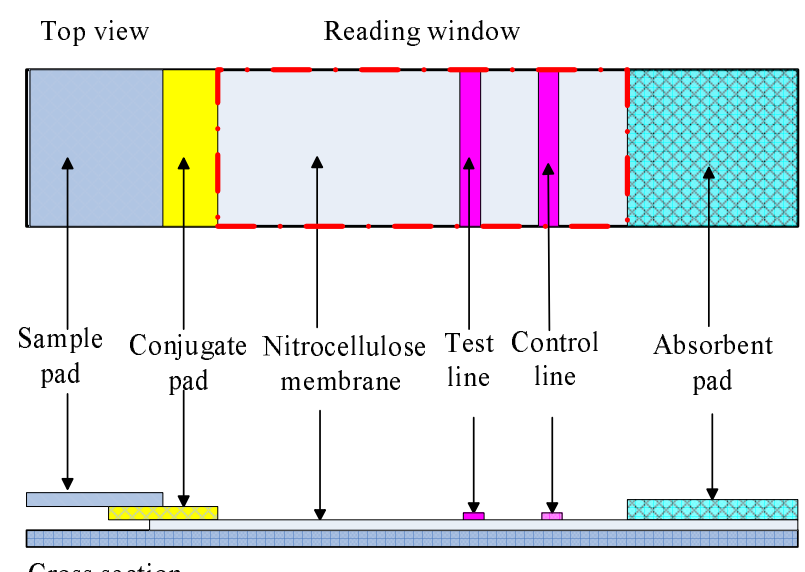

Cross section

Figure 1 Lateral flow immunoassay format.

For the LFIA device considered in this paper, we assume that there is only one single target analyte $A$ in the sample. Similar to the [37-39], the control line is not taken into account. According to the principle of the process of biochemical reactions, the signal pathway of the sandwich-type LFIA device can be conducted as follows [2.5, 37]:

(a) Once the sample flows through the conjugate pad, the analyte $A$ will interact with the labeled particle $P$ to form particle-analyte complexes $P A$,

$$
A+P \underset{k_{2}}{\stackrel{k_{1}}{\rightleftharpoons}} P A
$$

(b) The free analyte $A$ and the complexes $P A$ both flow through the membrane via the capillary action. Therefore, $A$ and $P A$ are going to interact with the $R$ which immobilized on the test line to form the complexes,

$$
\begin{gathered}
A+R \underset{k_{4}}{\stackrel{k_{3}}{\rightleftharpoons}} R A \\
P A+R \underset{k_{6}}{\stackrel{k_{5}}{\rightleftharpoons}} R P A
\end{gathered}
$$

(c) In addition, unbound labeled particle $P$ may interact with the complex $R A$ to form the complex $R P A$,

$$
P+R A \underset{k_{8}}{\stackrel{k_{7}}{\rightleftharpoons}} R P A
$$


It is worth highlighting that a nonlinear state-space model has recently been developed in [37-B9] for sandwich-type LFIA devices, where the established model accounts for the biochemical reaction process of LFIA system as well as the observation output. More specifically, the process of the concentration distribution of LFIA devices are described by the nonlinear state equations, and the available measurement signals are characterized by the observation equation including the measurement noises. In [37-39], however, it has been assumed that there is no time-delay in the process of LFIA reactions (四)-(四). Such an assumption is not really reasonable since the sample containing the target analytes needs time to flow from the sample pad along the nitrocellulose membrane encountering the detection zone via capillary action. In order to establish a more accurate model for the LFIA, the time-delays between the biochemical reactions are considered in this paper, and this constitutes one of the main contributions of the present research.

Let $x_{1}, x_{2}, x_{3}, x_{4}, x_{5}$ and $x_{6}$ be the concentration of $A, P, P A, R, R A$ and $R P A$, respectively. The reaction rates of the LFIA devices are defined as follows:

$$
\begin{aligned}
& v_{1}=k_{1} x_{1} x_{2}-k_{2} x_{3} \\
& v_{2}=\left(k_{3} x_{1} x_{4}-k_{4} x_{5}\right)\left(t-\tau_{1}\right) \\
& v_{3}=\left(k_{5} x_{3} x_{4}-k_{6} x_{6}\right)\left(t-\tau_{2}\right) \\
& v_{4}=\left(k_{7} x_{2} x_{5}-k_{8} x_{6}\right)\left(t-\tau_{3}\right)
\end{aligned}
$$

where $k_{1}, k_{3}, k_{5}, k_{7}$ and $k_{2}, k_{4}, k_{6}, k_{8}$ are, respectively, the association and dissociation rate constants, and $\tau_{1}, \tau_{2}, \tau_{3}$ are time delays which exist among the biochemical reactions (प)-(四). Then, the stoichiometrix for describing the biochemical reaction of the LFIA device is provided by

$$
S=\left[\begin{array}{cccc}
-1 & -1 & 0 & 0 \\
-1 & 0 & 0 & -1 \\
1 & 0 & -1 & 0 \\
0 & -1 & -1 & 0 \\
0 & 1 & 0 & -1 \\
0 & 0 & 1 & 1
\end{array}\right]
$$

Let $x=\left[x_{1}, x_{2}, \ldots, x_{6}\right]^{T}, V=\left[v_{1}, v_{2}, \ldots, v_{4}\right]^{T}, \tau=\left[\tau_{1}, \tau_{2}, \tau_{3}\right]^{T}$, and $\theta=\left[k_{1}, k_{2}, \ldots, k_{9}\right]^{T}$ stand for the parameters to be identified. The improved nonlinear state-space model with time-delay of the LFIA device is shown as follows:

$$
\begin{aligned}
x(k+1) & =f(x(k), \tau, \theta)+w(k) \\
y(k) & =g(x(k), \tau, \theta)+v(k)
\end{aligned}
$$

where $x(k)$ is the vector of state variables which are concentrations of antibodies, antigens or complex material; $y(k)$ is the observation experiment value; $f(x(k), \tau, \theta)=S V(x(k))$ is the nonlinear function to describe the transformation in the LFIA device; $V(x(k))$ stands for the vector of reaction rates [2.9]; $g(x(k), \tau, \theta)=k_{9}\left(x_{3}+x_{6}\right)$ is the observation function; $w(k)$ and $v(k)$ are the zero-mean independent Gaussian noises, respectively.

The objective of this paper is to jointly identify the parameters and states of the improved model (回)-(피) via the unscented Kalman filter method from the short available experiment data.

\section{Unscented Kalman Filter}

In this section, we introduce the UKF algorithm for estimating system states and parameters, see e.g. [5, [12, 24, 33] for more details.

The Kalman filter (KF) is developed as an optimal state estimator for a linear system. Based on the unscented transform (UT), the UKF algorithm is developed for nonlinear systems as a recursive state estimator. The unscented transform (UT) is a deterministic sampling technique, which utilizes a set of $2 n+1$ sample points (called "sigma points") to estimate the statistics characteristics of the transformed variable. Especially, the sigma points are generated deterministically from a priori mean and covariance of the states. Therefore, both the posterior mean and the covariance of UKF approach are computed 
directly from these $2 n+1$ sigma points by going through nonlinear transformation [5, [2, [24, 33.]. Note that the UKF can approximate the posterior mean and covariance for nonlinear systems without any linearization step, but can get second-order accuracy. However, the EKF algorithm can only obtain firstorder accuracy by using the linearization method. Therefore, the UKF represents a possible alternative to the extend Kalman filter (EKF), which requires evaluation of the Jacobians of the nonlinear functions in the state and output equations.

Consider the following system with nonlinear state and measurement functions:

$$
\begin{aligned}
x(k+1) & =f(x(k))+w(k) \\
y(k) & =g(x(k))+v(k)
\end{aligned}
$$

where $k$ is the time index, $x(k)$ is a state vector, $y(k)$ is the measurement vector, $w(k)$ and $v(k)$ are zero-mean white Gaussian noises with covariance matrices $Q_{k}$ and $R_{k}$ for the process and measurements, respectively. Here, $f: \mathbb{R}^{n} \rightarrow \mathbb{R}^{n}$ is a nonlinear state function and $g: \mathbb{R}^{n} \rightarrow \mathbb{R}^{r}$ is a nonlinear measurement function.

The procedure for implementing the UKF is given as follows:

1) Initialize in time step $k=0$ :

$$
\begin{aligned}
& \hat{x}_{0}=E\left[x_{0}\right] \\
& P_{x_{0}}=E\left[\left(x_{0}-\hat{x}_{0}\right)\left(x_{0}-\hat{x}_{0}\right)^{T}\right] \\
& \hat{x}_{0}^{a}=E\left[x_{0}^{a}\right]=\left[\begin{array}{lll}
\hat{x}_{0}^{T} & \hat{w}_{k}^{T} & \hat{v}_{k}^{T}
\end{array}\right] \\
& P_{x_{0}}^{a}=E\left[\left(x_{0}^{a}-\hat{x}_{0}^{a}\right)\left(x_{0}^{a}-\hat{x}_{0}^{a}\right)^{T}\right]=\operatorname{diag}\left(P_{x_{0}}, Q_{0}, R_{0}\right)
\end{aligned}
$$

For $k=1,2,3, \ldots$ compute

2) Calculate sigma points:

$$
\chi_{k-1}^{a}=\left[\hat{x}_{k-1}^{a} \hat{x}_{k-1}^{a}+\gamma\left(\sqrt{P_{k-1}}\right)_{i} \hat{x}_{k-1}^{a}-\gamma\left(\sqrt{P_{k-1}}\right)_{i}\right] \quad i=1, \ldots, 2 n
$$

where $n=n_{x}+n_{w}+n_{v}$ is the augmented dimension of $x^{a}, \gamma=\sqrt{n+\lambda}, \lambda=\alpha^{2}(n+\kappa)-n$.

3)Time update:

$$
\begin{aligned}
& \hat{\chi}_{i, k \mid k-1}^{(x)}=f\left(\chi_{i, k-1}^{(x)}, \chi_{i, k-1}^{(w)}\right) \\
& \hat{x}_{k \mid k-1}=\sum_{i=0}^{2 n} W_{i}^{(m)} \hat{\chi}_{i, k \mid k-1}^{(x)} \\
& P_{k \mid k-1}=\sum_{i=0}^{2 n} W_{i}^{(c)}\left\{\hat{\chi}_{i, k \mid k-1}^{(x)}-\hat{x}_{k \mid k-1}\right\}\left\{\hat{\chi}_{i, k \mid k-1}^{(x)}-\hat{x}_{k \mid k-1}\right\}^{T}
\end{aligned}
$$

where $W_{0}^{(c)}=\lambda /(n+\lambda)+1-\alpha^{2}+\beta, W_{0}^{(m)}=\lambda /(n+\lambda), W_{i}^{(c)}=W_{i}^{(m)}=1 / 2(n+\lambda), i=1, \ldots 2 n$. When the system meets the Gaussian noise assumptions, $\alpha, \beta$ and $\kappa$ in the UKF approach are typically set as $10^{-3}, 2$ and 0 respectively [34].

$$
\begin{aligned}
& \hat{y}_{i, k \mid k-1}=h\left(\chi_{i, k \mid k-1}, \chi_{i, k-1}^{(v)}\right) \\
& y_{k}^{-}=\sum_{i=0}^{2 n} W_{i}^{(m)} \hat{y}_{i, k \mid k-1}
\end{aligned}
$$

4) Measurement update:

$$
\begin{aligned}
& P_{y_{k}}=\sum_{i=0}^{2 n} W_{i}^{(c)}\left\{\hat{y}_{i, k \mid k-1}-y_{k}^{-}\right\}\left\{\hat{y}_{i, k \mid k-1}-y_{k}^{-}\right\}^{T} \\
& P_{x_{k} y_{k}}=\sum_{i=0}^{2 n} W_{i}^{(c)}\left\{\hat{\chi}_{i, k \mid k-1}^{(x)}-\hat{x}_{k \mid k-1}\right\}\left\{\hat{y}_{i, k \mid k-1}-y_{k}^{-}\right\}^{T}
\end{aligned}
$$




$$
\begin{aligned}
& \hat{x}_{k \mid k}=\hat{x}_{k \mid k-1}+K_{k / k}\left[y_{k}-y_{k}^{-}\right] \\
& K_{k / k}=P_{x_{k} y_{k}} P_{y_{k}}^{-1} \\
& P_{k / k}=P_{k / k-1}-K_{k / k} P_{y_{k}} K_{k / k}^{T}
\end{aligned}
$$

Remark 1. Note that UKF has advantages over the EKF in that there is no need to compute the Jacobian matrix and the accuracy may therefore be improved. It should be pointed out that the convergence of UKF has been discussed in [33] and the references therein.

\section{Results Using the UKF Approach and Discussion}

In this section, the unscented Kalman filter (UKF) is exploited to jointly estimate the states and parameters of the improved sandwich-type LFIA model with time-delay. The short time series data is used and shown in Fig. $\boldsymbol{\nabla}$. It should be mentioned that the short time series data, 45 equally spaced time points, is obtained when the sample flows over the nitrocellulose membrane within 11 minutes [37].
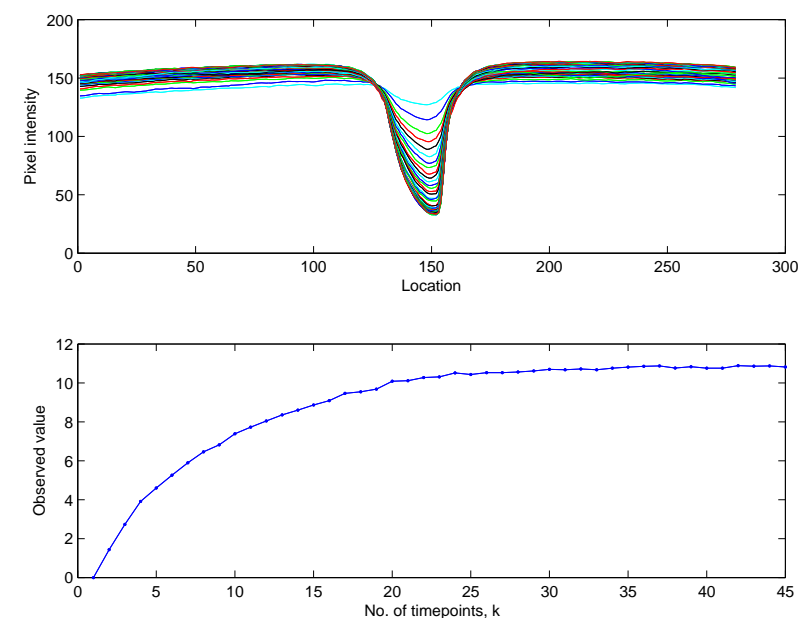

Figure 2 Upper part: The pixel intensity of the surface layer; Lower part: The measurement output of LFIA devices

The initial values of the state variables are set as

$$
x_{0}=[5,6.5,0,13,0,0]^{T}
$$

and the initial values of parameters are set as

$$
k_{0}=[0.03,0.0001,0.01,0.0001,0.04,0.0001,0.04,0.0001,2.2]^{T} .
$$

In the improved LFIA model with time-delay, we assume that the time-delay $\tau_{2}$ equals to the time-delay $\tau_{3}$, and is larger than the $\tau_{1}$ according to the biochemical reactions of the sandwich-type LFIA. Then, the UKF algorithm is applied to jointly identify parameters and state variables of the LFIA devices. The identified parameters in the nonlinear model and the system state variables that depicted in the form of time series are given in Fig. B and Fig. 田.

Similar to the [37], three experiments by modifying the concentration of the target analyte $A$ are made to further evaluate the UKF method for inferring the nonlinear LFIA state-space models with time-delay. The results are given in Fig. 1 . The red line shows the observed signal from the experiments, and the blue line shows the predicted value by the UKF algorithm. Especially, the error ratio in percentage [22,31,37] is exploited to evaluate the model quality in a quantitative way, which shows the modeling errors between the experiment values and the predicted value.

$$
\text { Error ratio }=100 \times \frac{1}{l} \sum_{c=1}^{l}\left[\sqrt{\frac{\sum_{k=1}^{s}\left(y_{c k}-\hat{y}_{c k}\right)^{2}}{\sum_{k=1}^{s}\left(y_{c k}\right)^{2}}}\right] \%
$$


Zeng N Y, et al. Sci China Inf Sci January 2016 Vol. xx xxxxxx:7

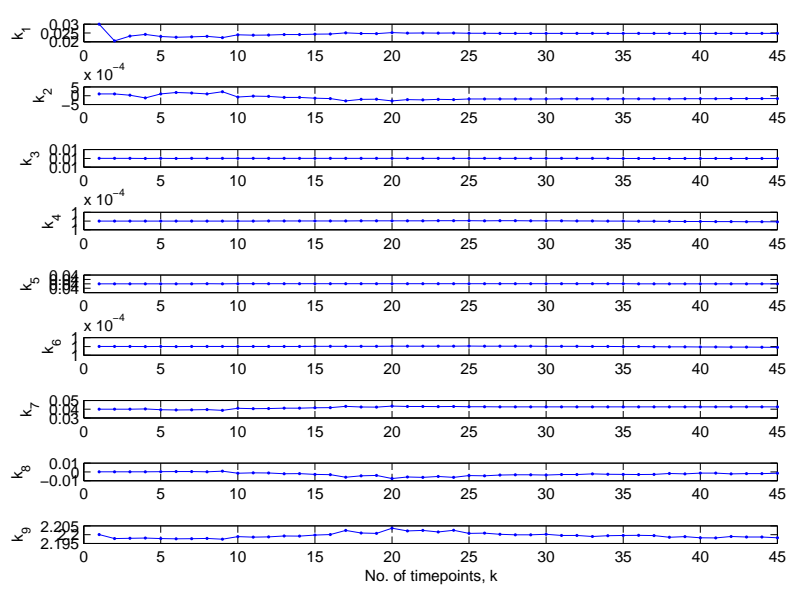

Figure 3 The identified time series of parameters $k_{1}, k_{2}, k_{3}, k_{4}, k_{5}, k_{6}, k_{7}, k_{8}, k_{9}$.

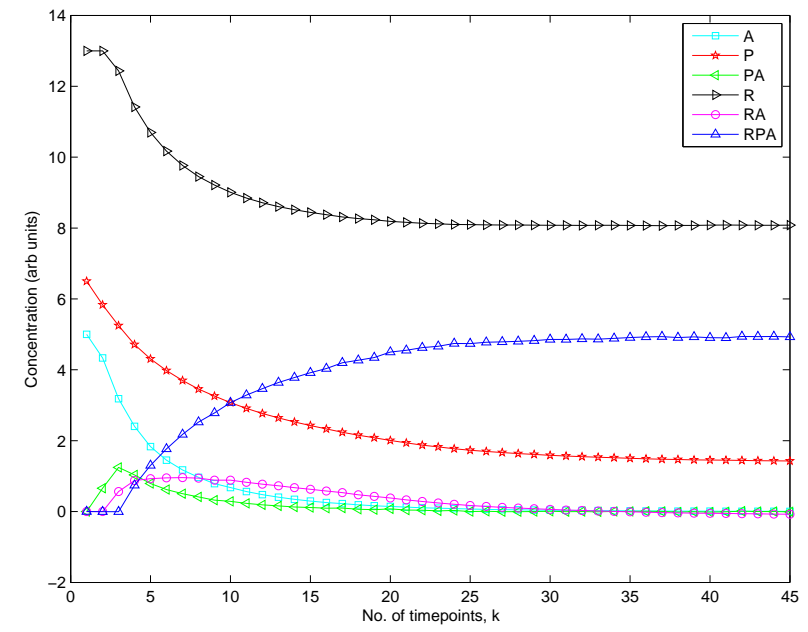

Figure 4 The identified time series of states $A, P, P A, R, R A, R P A$.

Table 1 Error ratio in percentage for evaluating the model quality

\begin{tabular}{|c|c|c|c|}
\hline \hline Experiment & 1 & 2 & 3 \\
\hline Error ratio & $1.55 \%$ & $2.41 \%$ & $3.72 \%$ \\
\hline \hline
\end{tabular}

where $l$ is the dimension of the observations ( $l=1$ in this paper); $s$ is the length of observations, and $y_{c k}$ is the experiment value for $c$ th observation at the $k$ th time point. The results are provided in Table $\mathbf{m}$. It is noticed that the error ratio of the UKF approach for modeling the LFIA with time-delays through only 45 time points (images) is generally satisfactory.

\section{Conclusion}

In this paper, we have applied the unscented Kalman filter (UKF) algorithm to model the lateral flow immunoassay via short but available time series data. The model for the LFIA is considered as a nonlinear state-space model, which includes the equations for describing the biochemical reaction process of LFIA system and the observation output. Note that the time-delays which exist among the biochemical reactions are included in the established model. In the end, the UKF approach is successfully exploited to identify not only the model parameters but also states of the improved nonlinear state-space model 


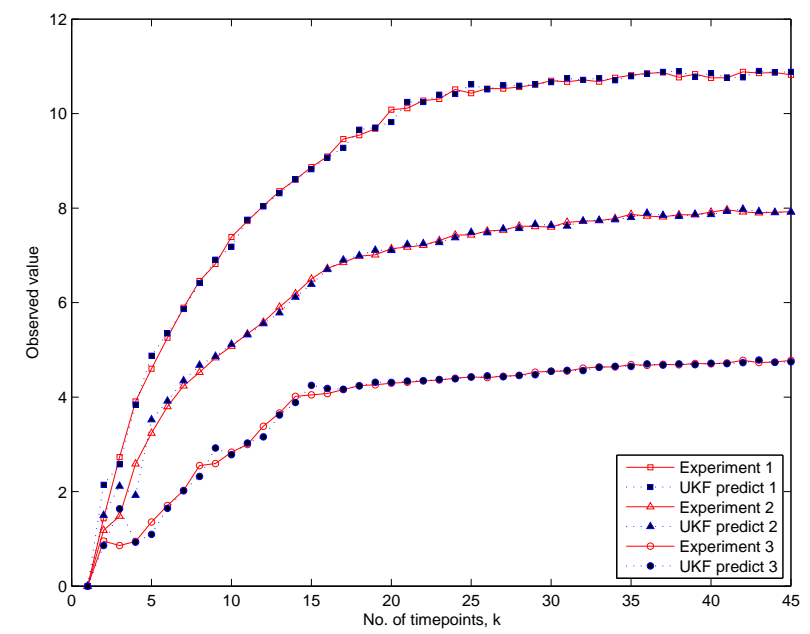

Figure 5 The observed value predicted by UKF method and observed value from the experiment.

with time-delay simultaneously. Experiment results have shown the effectiveness of the UKF approach for modelling the lateral flow immunoassay.

In the near feature, our research topics will focus on the analysis of LFIA system with more complicated

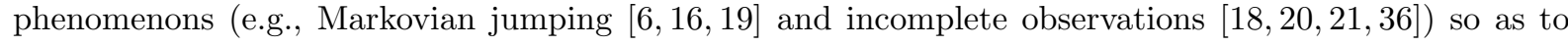
comprehensively simulate the biochemical reaction network of LFIA system.

Acknowledgements This work was supported in part by the Natural Science Foundation of China under Grant 61403319, in part by the Fujian Natural Science Foundation under Grant 2015J05131, in part by the Fujian Provincial Key Laboratory of Eco-Industrial Green Technology, and in part by the Fundamental Research Funds for the Central Universities.

Conflict of interest The authors declare that they have no conflict of interest.

\section{References}

1 Chuang L, Hwang J, Chang H, et al. Rapid and simple quantitative measurement of a-fetoprotein by combining immunochromatographic strip test and artificial neural network image analysis system. Cli Chim Acta, 2004, 348: 8793

2 Du M, Fang Z, Fei H. Application of photoelectric sensor to quantitative determination of immunochro-matographic assay strip. Chin J Sci Instr, 2005, 36(7): 671-673

3 Faulstich K, Gruler R, Eberhard M, et al. Developing rapid mobile POC systems. Part 1:Devices and applications for lateral-flow immunodiagnostics. IVD Tech, 2007, 13(6): 47-53

4 Gillespie J, Gannot G, Tangrea M, et al. Molecular profiling of cancer. Toxicol Pathol, 2004, 32: 67-71

5 Giannitrapani A, Ceccarelli N, Scortecci F, et al. Comparison of EKF and UKF for spacecraft localization via angle measurements. IEEE T Aero Elec Sys, 2011, 47(1): 75-84

6 Hou N, Dong H, Wang Z, et al. Non-fragile state estimation for discrete Markovian jumping neural networks. Neurocomputing, 2016, 179: 238-245

7 Huang L, Zhang Y, Xie C, et al. Research of reflectance photometer based on optical absorption. Optik, 2010, 121(19): $1725-1728$

8 Huang S, Wei H and Lee Y, One-step immunochro-matographic assay for the detection of Staphylococcus aureus. Food Control, 2007, 18(8): 893-897

9 Kaur J, Singh K, Boro R, et al. Immunochromatographic dipstick assay format using gold nanoparticles labeled protein-hapten conjugate for the detection of atrazine. Environ Sci Technol, 2007, 41(14): 5028-5036

10 Kolosova A, Saeger S, Sibanda L, et al. Development of a colloidal gold-based lateral-flow immunoassay for the rapid simultaneous detection of zearalenone and deoxynivalenol. Anal Bioanal Chem, 2007, 389: 2103-2107

11 Laderman E, Whitworth E, Dumaual E, et al. Rapid, sensitive, and specific lateral-flow immunochromatographic point-of-care device for detection of herpes simplex virus type 2-specific immunoglobulin G antibodies in serum and whole blood. Clin Vaccine Immunol, 2008, 5: 159-163

12 Lei M, Han C. Sequential nonlinear tracking using UKF and raw range-rate measurements. IEEE T Aero Elec Sys, 2007, 43(1): 239-250

13 Li D, Wei S, Yang H, et al. A sensitive immunochromatographic assay using colloidal gold-antibody probe for rapid detection of pharmaceutical indomethacin in water samples. Biosens Bioelectron, 2009, 24(7): 2277-2280

14 Li J, Ouellette A, Giovangrandi L, et al. Optical scanner for immunoassays with up-converting phosphorescent labels. IEEE T Bio-med Eng, 2008, 55(5): 1560-1571 
Zeng N Y, et al. Sci China Inf Sci January 2016 Vol. xx xxxxxx:9

15 Li Y, Zeng N, Du M. Study on the methodology of quantitative gold immunochromatographic strip assay. Proc. of the 2010 International Workshop on Intelligent Systems and Application, 2010, 182-185

16 Liu Y, Liu W, Obaid M, et al. Exponential stability of Markovian jumping Cohen-Grossberg neural networks with mixed mode-dependent time-delays. Neurocomputing, 2016, 177: 409-415

17 Li W, Wei G, Han F, et al. Weighted average consensus-based unscented Kalman filtering. IEEE T Cybernetics, 2016 , 46(2): $558-567$

18 Liu S, Wei G, Song Y, et al. Error-constrained reliable tracking control for discrete time-varying systems subject to quantization effects. Neurocomputing, 2016, 174: 897-905

$19 \mathrm{Li} \mathrm{Q}$, Shen B, Liu Y, et al. Event-triggered H infinity state estimation for discrete-time stochastic genetic regulatory networks with Markovian jumping parameters and time-varying delays. Neurocomputing, 2016, 174: 912-920

20 Luo Y, Wei G, Liu Y, et al. Reliable H-infinity state estimation for 2-D discrete systems with infinite distributed delays and incomplete observations. Int J Gen Syst, 2015, 44(2): 155-168

21 Liu Y, Alsaadi F, Yin X, et al. Robust H-infinity filtering for discrete nonlinear delayed stochastic systems with missing measurements and randomly occurring nonlinearities. Int J Gen Syst, 2015, 44(2): 169-181

22 Ljung L. System Identification: Theory for the User, 2nd ed. Upper Saddle River, NJ: Prentice-Hall, 1999

23 Lundblad R, Wagner P. The potential of proteomics in developing diagnostics. IVD Tech, 2005, 3: 20-22

24 Meng W, Chen X, Li C, et al. UKF-based iterative channel estimation using two-dimensional block spread coding for uplink transmission in multicarrier CDMA networks. IEEE T Veh Technol, 2013, 62(9): 4444-4457

25 Qian S, Haim H. A mathematical model of lateral flow bioreactions applied to sandwich assays. Anal Biochem, 2003, 322(1): 89-98

26 Qian S, Haim H. Analysis of lateral flow biodetectors: competitive format. Anal Biochem, 2004, 326(2): 211-224

27 Quach M, Brunel N, d'Alché-Buc F. Estimating parameters and hidden variables in non-linear state-space models based on ODEs for biological networks inference. Bioinformatics, 2007, 23(23): 3209-3216

28 Raphael C, Harley Y. Lateral Flow Immunoassay. New York: Humana Press, 2008

29 Sun X, Jin L, Xiong M. Extended Kalman filter for estimation of parameters in nonlinear state-space models of biochemical networks. PLoS ONE, 2008, 3(11): e3758

30 Tanaka R, Yuhi T, Nagatani N, et al. A novel enhancement assay for immunochromatographic test strips using gold nanoparticles. Anal Bioanal Chem, 2006, 385(8): 1414-1420

31 Wang Z, Yang F, Ho D, et al. Stochastic dynamic modeling of short gene expression time series data. IEEE T NanoBiosci, 2008, 7(1): 44-55

32 Wu N, Li B, Wang H, et al. Distributed cooperative localization based on Gaussian message passing on factor graph in wireless networks. Sci China-Inf Sci, 2015, 58: 042305

33 Xiong K, Chan C, Zhang H. Detection of satellite attitude sensor faults using the UKF. IEEE T Aero Elec Sys, 2007, 43(2): 480-491

$34 \mathrm{Xu} \mathrm{B}$, Zhu H, Ji W. State estimation of bearingless permanent magnet synchronous motor using improved UKF. Proceedings of the 31st Chinese Control Conference, 2012, 4430-4433

35 Xue M, Li X, Fu L, et al. X-ray pulsar-based navigation using pulse phase and Doppler frequency measurements. Sci China-Inf Sci, 2015, 58: 122202

$36 \mathrm{Yu}$ Y, Dong H, Wang Z, et al. Design of non-fragile state estimators for discrete time-delayed neural networks with parameter uncertainties. Neucomputing, 2016, 182: 18-24

37 Zeng N, Wang Z, Li Y, et al. Inference of nonlinear state-space models for sandwich-type lateral flow immunoassay using extended Kalman filtering. IEEE T Bio-med Eng, 2011, 58(7): 1959-1966

38 Zeng N, Wang Z, Li Y, et al. Identification of nonlinear lateral flow immunoassay state-space models via particle filter approach. IEEE T Nanotechnol, 2012, 11(2): 321-327

39 Zeng N, Wang Z, Li Y, et al. A hybrid EKF and switching PSO algorithm for joint state and parameter estimation of lateral flow immunoassay models. IEEE ACM T Comput Bi, 2012, 9(2): 321-329

40 Zeng N, Wang Z, Li Y, et al. Time series modeling of nano-gold immunochromatographic assay via expectation maximization algorithm. IEEE T Bio-med Eng, 2013, 60(12): 3418-3424

41 Zeng N, Hung Y, Li Y, et al. A novel switching local evolutionary PSO for quantitative analysis of lateral flow immunoassay. Expert Syst Appl, 2014, 41(4): 1708-1715

42 Zeng N, Wang Z, Zineddin B, et al. Image-based quantitative analysis of gold immunochromatographic strip via cellular neural network approach. IEEE T Med Imaging, 2014, 33(5): 1129-1136

43 Zeng N, Wang Z, Zhang H, et al. A novel switching delayed PSO algorithm for estimating unknown parameters of lateral flow immunoassay. Cogn Comput, 2016, 8(2): 143-152

44 Zhang G, Wang X, Zhi A, et al. Development of a lateral flow immunoassay strip for screening of sulfamonomethoxine residues. Food Addit Contam A, 2008, 25(4): 413-423

45 Zhu J, Chen W, Lu Y, et al. Development of an immunochromatographic assay for the rapid detection of bromoxynil in water. Environ Pollut, 2008, 156(1): 136-142 